\title{
鸟类和蚂蚁对桃金娘种子传播的初步研究
}

\author{
韦明思 ${ }^{1}$ 陈章和 ${ }^{*}$ 任 海 $^{2}$ 邹发生 ${ }^{3}$ 殷祚云 $^{24}$ \\ 1 (华南师范大学生命科学学院, 广州 510631) \\ 2 (中国科学院华南植物园, 广州 510650) \\ 3 (华南濒危动物研究所, 广州 510260) \\ 4 (广东省林业科学研究院, 广州 510520)
}

摘要 : 在中国科学院鹤山丘陵综合开放试验站观察研究了乌类和蚂蚁对桃金娘 (Rhodomyrtus tomentosa) 种子的传 播。据野外观察和网捕法所捕鸟的情况可知 红耳鹅 (Pycnonotus jocosus) 和白头軒 ( $P$. sinensis) 两种鸟传播桃金

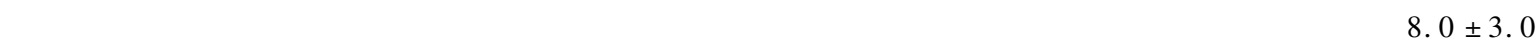
粒/只和 $10.0 \pm 2.0$ 粒/只。在显微镜下观察经过鸟消化道的桃金娘种子都没有破损。传播桃金娘种子的蚂蚁有 全异巨首蚁 (Pheidologetion diversus) 和梅花山大头蚁 (Pheidole meihuashanensis)。它们传播的最远距离分别为 $4 \mathrm{~m}$ 和 $5 \mathrm{~m}$ 平均为 $1.1 \pm 0.09 \mathrm{~m}$ 和 $1.3 \pm 0.07 \mathrm{~m}$ 。全异巨首蚁占样地蚂蚁个体总数的 $60.0 \%$ 梅花山大头蚁占个体总 数的 $8.1 \%$; 余下的为其他种蚂蚁。每个全异巨首蚁蚁巢平均咜藏桃金娘种子 $195.0 \pm 82.9$ 粒 其中 $73.2 \%$ 是完好 种子。每个梅花山大头蚁蚁巢平均贮藏 $28.8 \pm 11.4$ 粒种子, 全部是完好种子。林窗、闲置的空旷地、植物群落交 错带都有桃金娘实生苗。在阳光充足的全异巨首蚁和梅花山大头蚁蚁巢边也有桃金娘实生苗的存在。

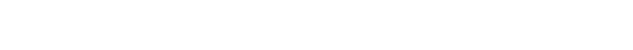

中图分类号 : Q948 文献标识码 : A 文章编号 : 1005-0094(2004)05-0494-07

\section{Seed dispersal of the pioneer shrub Rhodomyrtus tomentosa by frugivorous birds and ants}

WEI Ming-Si ${ }^{1}$, CHEN Zhang-He ${ }^{1 *}$, REN Hai $^{2}$, ZOU Fa-Sheng ${ }^{3}$, YIN Zuo-Yun ${ }^{2}{ }^{4}$

1 College of Life Science, South China Normal University, Guangzhou 510631

2 South China Botanical Garden, Chinese Academy of Sciences, Guangzhou 510650

3 South China Institute of Endangered Animals , Guangzhou 510260

4 Guangdong Research Institute of Forestry, Guangzhou 510520

\begin{abstract}
Field work was done in Heshan city , Guangdong Province on seed dispersal of Rhodomyrtus tomentosa by frugivorous birds and ants. $R$. tomentosa is a very common pioneer species in the South China , and its pulp is rich in glucide and fat. Pycnonotus jocosus and $P$. sinensis were the birds that ate $R$. tomentosa fruits and dispersed the seeds in the study area , and the later was more than the former in number during the observation period. The seed number of this shrub in the feces of $P$. jocosus and $P$. sinensis caught in mist nets were $8.0 \pm 3.0$ and $10.0 \pm 2.0$, respectively. Two species of ants ,Pheidologetion diversus and Paratrechina flavipes, were observed eating $R$. tomentosa pulp. Pheidologetion diversus and another ant species Pheidole meihuashanensis were observed carrying the seeds. The longest distances of movement by Pheidologetion diversus and Pheidole meihuashanensis were $4 \mathrm{~m}$ and $5 \mathrm{~m}$, respectively , and the mean distances of dispersal by these agents were $1.1 \pm 0.09 \mathrm{~m}$ and $1.3 \pm 0.07 \mathrm{~m}$, respectively. 657 ants were collected in the study area by pitfall trapping, comprising 394 Pheidologetion diversus (60.0\%) , 144 Paratrechina flavipes (21.9\%) ,53 Pheidole meihuashanensis ( $8.1 \%$ ) and other species. The average number of stored seeds in a Pheidologetion diversus nest was 195. $0 \pm 82.9$, among which $73.2 \%$ were intact. And the average number of stored seeds in a Pheidole meihuashanensis nest was $28.8 \pm 11.4$, all of which were intact. The seedlings of $R$. tomentosa could be found at canopy gaps , on bare land and at the forest edge. There were also many seedlings near ant's nests wherever sunlight was
\end{abstract}


high.

Key words : Pycnonotus jocosus ; Pycnonotus sinensis ; frugivorous birds ; mist net ; myrmecochory

植物是固着的生物，自身传播种子的能力有限。 如果种子落在母树下,会有许多不利，如与母树竞 争资源、母树附近的密度制约性死亡、幼苗之间的竞 争等 (Howe \& Smallwood, 1982; McCall et al. , 1989 ; Peres et al. ,1997 ; Hulme , 1998)。所以在进 化上能存活下来的植物都存在某种机制使种子远离 母树 到达安全地，以便有更高的萌发率和幼苗存活 率。传播种子的媒介有许多种，许多种子的传播是 依靠动物的活动来完成, 其中鸟类是一类重要的传 播者,而蚂蚁也是传播种子的一个非常大的群体 (Edmund，2000)。食果鸟和蚂蚁对种子的传播，国 外做了大量的研究 (Cruz, 1981 ; David \& Devon, 2000 ; Jennifer et al. , 2001 ; Ron \& Thomas , 2002； Jose et al. ,2003)。我国在这方面的研究近几年也 已经开始（肖来云和普正和，1994; 黄双全和王孝 民，2000 张智英等，2001; 王直军等，2002; 鲁长虎， 2003 )。

桃金娘 (Rhodomyrtus tomentosa) 是华南地区退 化荒坡植被自然恢复过程中很常见的先锋灌木，对 华南地区退化山坡的植被恢复起着重要的作用 (中 国科学院华南植物研究所,1956)。桃金娘不能在 透光率为 $32 \%$ 以下的林冠下正常生长并完成更新 过程, 说明其幼树生长需充足的阳光, 是迹地或空旷 地上更新的阳性树种 ( 任海等, 1997)。种群的迁入 有许多原因，但种源的迁入是先决条件。桃金娘种 子为何能够迁入到迹地或空旷地, 形成实生苗? 其 果实较大, 肉质, 富含糖类和脂肪, 可能是对动物传 播种子的适应。因此，研究动物对桃金娘果实取食 和对种子传播的规律，深入了解桃金娘的扩散和分 布特点，对华南地区的植被恢复有重要意义。但有 关动物对桃金娘种子传播的研究至今未见报道。

\section{1 材料方法}

\section{1 样地和材料}

研究地点位于中国科学院鹤山丘陵综合开放试 验站。鹤山丘陵站位于广东省中部的鹤山市, 东经 $112^{\circ} 54^{\prime}$, 北纬 $22^{\circ} 41^{\prime}$, 属南亚热带季风气候。年平 均气温 $21.7^{\circ} \mathrm{C}$, 极端高温 $37.5^{\circ} \mathrm{C}$, 极端低温 $0^{\circ} \mathrm{C} ;$;年 均太阳辐射 $4350.5 \mathrm{MJ} \mathrm{m}^{-2} \mathrm{a}^{-1}$;年均 $\geqslant 10^{\circ} \mathrm{C}$ 有效积
温 $7597^{\circ} \mathrm{C}$; 年均降雨量 $1800 \mathrm{~mm}$, 有明显的干湿季 之分，干季为 10 月至翌年 2 月，湿季为 3-9 月;年 均蒸发量 $1638.8 \mathrm{~mm}$, 丘陵地土壤为赤红壤(林永标 等 2003)。地带性植被为亚热带季风常绿润叶林, 因人为干扰, 许多地方退化为亚热带草坡 (彭少麟, 1996 )。研究样地为南坡, 坡度约为 $10^{\circ}$ 。植物除了 优势种桃金娘外,还有芒萁 (Dicranopteris dichoto$\mathrm{ma}$ )、乌毛烣 (Blechnum orientale)、弓果㯟 (Cyrtococcum patens)、地稔 (Melastoma dodecendrum)、岗松 (Baeckea frutescens) 、三叉苦 (Evodia lepta)、梅叶冬 青 (Ilex asprella)、山黄麻 (Trema orientlis)、野牡丹 (Melastoma candidum)、石斑木 (Rhaphiolepis indi$c a$ )、黑面神 (Breynia fruitcosa)、山鸡椒 (Litsea cube$b a) 、$ 玉叶金花 (Mussaenda pubescens)、酸藤子 ( Embelia laeta $)$ 等。

桃金娘为桃金娘科、桃金娘属的阳生性灌木，高 1-2 $\mathrm{m}$ 花期 4-6 月, 果实成熟期 7-9 月。浆果卵 状壶形, 长 $1.5-2.0 \mathrm{~cm}$, 宽 $1-1.5 \mathrm{~cm}$,成熟时紫黑 色, 果肉紫红色, 肉质多汁, 可鲜食。每一浆果鲜重 为 $1.4 \pm 0.09 \mathrm{~g}$ 。桃金娘鲜果的主要成分: 水占 $62.99 \%$ 总糖占 $11.25 \%$ ，蛋白质占 $1.82 \%$ ，脂肪占 $11.20 \%$ (刘芳和邓毓芳, 1997)。每一浆果有种子 $53.0 \pm 7.0$ 粒。种子扁圆形, 直径为 $1.4 \pm 0.2 \mathrm{~mm}$, 厚为 $0.4 \pm 0.1 \mathrm{~mm}$ 。种子的千粒重为 $10.0 \pm 1.2 \mathrm{~g}$ 。 桃金娘广泛分布于我国的台湾、广西、云南、贵州、湖 南和广东; 中南半岛、菲律宾、日本、印度、斯里兰卡、 马来西亚和印度尼西亚也有分布 (中国科学院中国 植物志编辑委员会, 1984)。

\section{2 研究方法}

\subsection{1 乌类对桃金娘果实的取食和对种子的传播}

选取 $10 \mathrm{~m} \times 10 \mathrm{~m}$ 的固定样方,在 2003 年果实 成熟盛期的 8 月 18 日至 8 月 22 日，用望远镜在隐 蔽处观察鸟类对样方内桃金娘种子的取食。记录来 样地访问的鸟的种类、个体数、频次及取食行为。连 续观察 $5 \mathrm{~d}$, 每天观察时间为 $630-1230$ 和 1430 -1830 。

网捕法捕鸟参照鲁长虎 (2003) 的方法。在 2003 年果实成熟盛期的 8 月 18 日至 8 月 22 日，在 样地共张开 6 个雾网 $(13 \mathrm{~m} \times 3 \mathrm{~m})$, 每天张网时间 
为 $630-1830$, 连续 $5 \mathrm{~d}$ 。记录捕到的鸟的种类。 把捕到的鸟放入布袋中，收集鸟粪中的桃金娘种子。 带回实验室笼养 $3 \mathrm{~d}$, 喂食桃金娘果实, 观察鸟的取 食情况，收集粪便中的桃金娘种子。然后在显微镜 下观察收集到的桃金娘种子有无破损。

\subsection{2 蚂蚁对桃金娘种子的搬运}

从 2003 年 8 月 23 日至 8 月 29 日进行蚂蚁搬 运桃金娘种子的观察: (1) 在样地里的桃金娘树下, 观察蚂蚁对调落的桃金娘果实和种子的取食和搬运 行为、测量蚂蚁搬运种子的距离、标记埋藏的地点。 观察时间为 8:00-18:00, 连续进行两天。采集取 食桃金娘果肉和搬运其种子的蚂蚁，装入盛有 $75 \%$ 的乙醇的小瓶中, 带回实验室鉴定。(2) 在实验样 地随机放 20 颗新鲜成熟的桃金娘果实。其中 10 颗 用铒子弄破表皮, 像鸟取食时啄破表皮一样; 另 10 颗没有弄破表皮, 是完好的果实。观察、测量、标记 的内容同上。同样把蚂蚁带回实验室鉴定。观察时 间为 $800-1800$, 连续进行两天, 重复两次。

样地蚂蚁调查于 2003 年 8 月 30 日至 9 月 4 日 进行。将观察鸟类的 $10 \mathrm{~m} \times 10 \mathrm{~m}$ 的样方划分成 16 个小样方 $(2.5 \mathrm{~m} \times 2.5 \mathrm{~m})$ ，每个小样方随机埋入口 径为 $7 \mathrm{~cm}$, 体积为 $180 \mathrm{~mL}$ 的一次性塑料杯一个, 杯 口与地面齐平 杯四周用泥土填平, 杯中放入 $50 \mathrm{~mL}$ 的 $5 \%$ 的乙醛, 放置 $48 \mathrm{~h}$ 。将捕到的蚂蚁装入盛有 $75 \%$ 乙醇的小瓶中保存, 带回实验室鉴定。记录和 统计捕到蚂蚁的种类和数量。实验时间为 $8: 00-$ 1800 , 连续进行两天, 重复两次。

2003 年 10 月 15 日对标记的蚁巢进行桃金娘 种子的调查, 每种蚁调查 5 个巢。调查种子放置的 位置, 记录和统计完好种子和受损种子数目。

\subsection{3 桃金娘实生苗的生长与分布情况}

2004 年 3 月调查闲置的空旷地、林窗、植物群 落交错带、林下、蚁巢旁边的桃金娘实生苗情况。

\section{2 结果与分析}

\section{1 来访鸟类及其取食行为}

在样地里观察到大山雀 (Parus major)、暗绿绣 眼 (Zosterops japonica simplex)、红耳軾 (Pycnonotus jocosus)、白头軒 ( $P$. sinensis)、长尾缝叶莺( Orthotomus sutorius longicaudus)、四声杜鹃 (Cuculus micropterus)、大杜鹃 (C. canorus)、原鸡 (Gallus gallus)、 金腰燕 (Hirundo daurica) 和斑头鸺留 (Glaucidium cuculoides) 共 10 种鸟, 而来样方里寻食的鸟类只有 4 种 :大山雀、暗绿绣眼、红耳軾、白头軒。其中只观 察到红耳鹎和白头軒采食桃金娘果实和种子。

取食时一般是白头軒一边鸣叫一边飞向有成熟 果实的枝条, 然后落在枝条上, 先豚破紫黑色成熟果 实的果皮 然后豚食果肉和种子。往往是吃了一颗 果实的一部分的时候，果实就掉落到地上。白头軾 取食后一般喜欢飞到较空旷的高处去休息,例如高 压线上、植物群落交错带、林窗旁的高树上, 并将粪 便撒落在这些地方。红耳鸡的取食行为与白头鸡相 似。来样地里采食桃金娘果实和种子的白头軒比红 耳䠋数量多,一天中 $630-830$ 和 $16: 30-18: 30$ 这两个时间段来样地采食的食果鸟数量最多 (表 $1)$ 。

在样地里用雾网捕到的鸟类共有 5 种。它们是 大山雀、暗绿绣眼、红耳鸺、白头鸺、长尾缝叶莺。在 $5 \mathrm{~d}$ 时间里捕到的红耳鹎和白头鹎分别为 5 只和 9 只;每只红耳鸺和白头鸺排出的桃金娘种子分别是 $8.0 \pm 3.0$ 粒和 $10.0 \pm 2.0$ 粒。室内喂食时也只有 这两种鸟取食桃金娘果实和种子。这和野外观察到 的情况一致。在显微镜下观察粪便中的桃金娘种子 并没有破损。由此可知 :对桃金娘的种子有传播作 用的是红耳殅和白头軾, 其中白头軒起的传播作用 比红耳䠋的大。

\section{2 蚂蚁的受食和搬运行为}

受食桃金娘果肉的蚂蚁有两种:全异巨首蚁 (Pheidologetion diversus) 和黄立毛蚁 (Paratrechina flavipes)。而搬运桃金娘种子的蚂蚁也是两种: 全异 巨首蚁和梅花山大头蚁 (Pheidole meihuashanensis)。

全异巨首蚁的工蚁单独搜寻食物，遇到表皮已 破 (不管是被鸟类豚破表皮调落还是人工弄破表 皮) 的桃金娘果实后就回巢召集同伴前来取食。它 们原地吃桃金娘的果肉，从表皮已破的地方开始。 在取食的过程中，一部分工蚁从附近搬运细泥把浆 果全部盖住, 只留一个小口供取食的时候进出。而 在这期间另一部分蚂蚁一直在里面取食, 直到把果 肉全部吃完, 吃完一颗果肉需要 $90.0 \pm 13.0 \mathrm{~min}$ 。 吃完果肉后全异巨首蚁并不在原地吃桃金娘的种 子, 而是一只蚂蚁搬运一粒种子运往巢里。它们的 搬运速度极快，搬运 $1 \mathrm{~m}$ 的距离仅需要 $5.0 \pm 2.0$ min。搬到蚁窝后就把种子丢弃在巢口, 也有的种子 被丢弃在途中, 占搬运种子数的 $21 \%$ 。搬运距离可 
表 1 各个时间段取食桃金娘果实鸟类的数量

Table 1 Number of frugivorous birds visiting on Rhodomyrtus tomentosa in different time period

\begin{tabular}{|c|c|c|c|c|c|c|c|}
\hline & \multirow{2}{*}{$\begin{array}{c}\text { 观察日期 } \\
\text { Observing date }\end{array}$} & \multicolumn{5}{|c|}{$\begin{array}{c}\text { 各个时间段取食鸟类的数量 } \\
\text { Number of visiting birds in different time period }\end{array}$} & \multirow{2}{*}{$\begin{array}{l}\text { 访问鸟的总数 } \\
\text { Total }\end{array}$} \\
\hline & & $630-830$ & $830-1030$ & $1030-1230$ & $1430-1630$ & $1630-1830$ & \\
\hline 白头鹎 & $2003-08-18$ & 2 & 1 & 0 & 0 & 4 & 7 \\
\hline \multirow[t]{2}{*}{ Pycnonotus sinensis } & 2003-08-19 & 2 & 2 & 1 & 1 & 3 & 9 \\
\hline & $2003-08-20$ & 3 & 0 & 0 & 0 & 2 & 5 \\
\hline 访问鸟的总数 Total & & 12 & 5 & 2 & 2 & 14 & 35 \\
\hline 红耳鹎 & $2003-08-18$ & 2 & 1 & 0 & 0 & 0 & 3 \\
\hline \multirow[t]{2}{*}{ Pycnonotus jocosus } & 2003-08-19 & 1 & 0 & 0 & 1 & 3 & 5 \\
\hline & $2003-08-20$ & 2 & 0 & 1 & 0 & 1 & 4 \\
\hline
\end{tabular}

长达 $4 \mathrm{~m}$, 平均距离是 $1.1 \pm 0.09 \mathrm{~m}$ 。每颗果实均有 $7.0 \pm 2.0$ 粒种子没有被搬走。留在原地的种子与 被搬走的种子在形态上没有什么明显区别。

黄立毛蚁也是单独受食，遇到表皮已破的果实 后, 招呼同伴列队前往, 在原地取食。它们只吃果 肉, 吃完一颗果肉需要的时间为 $2.0 \pm 0.4 \mathrm{~h}$ 。吃完 后把果皮和种子丢弃在原地,并不把种子搬运走。 梅花山大头蚁不吃果肉, 对种子也没有伤害, 而是仅 仅搬运丢弃在地上的种子。这种丢弃的种子大多是 黄立毛蚁吃完果肉遗留下来的, 而不是全异巨首蚁 留在原地被泥土盖住的种子。梅花山大头蚁一次搬 运 1 粒种子。搬运 $1 \mathrm{~m}$ 的距离需要的时间为 $3.0 \pm$ $1.0 \mathrm{~min}$ 搬运的距离可长达 $5 \mathrm{~m}$, 平均距离为 $1.3 \pm$ $0.07 \mathrm{~m}$ 。搬到蚁窝后就把种子丢弃在巢口,但有 18\%的种子被丢弃在途中。它们也没有把黄立毛蚁 丢弃的种子全部搬走，每颗桃金娘果实留在原地的 种子数为 $27.0 \pm 6.0$ 粒。

全异巨首蚁既取食桃金娘的果肉也搬运其种 子。黄立毛蚁取食桃金娘的果肉, 但没有搬运桃金 娘种子 其留下的种子被梅花山大头蚁搬走, 起到了 间接搬运种子的作用。这些蚂蚁在观察当天取食表 皮已破的桃金娘果实,并搬运其种子，而没有取食表 皮完好的桃金娘果实,但是第二天早上这些没有被 取食的果实也不见了, 原地只留一点果皮和少量的 种子。这表明 红耳唡和白头鹅在取食过程中, 把桃
金娘的果实豚掉在地上,被豚破表皮的果实的果肉 更容易被蚂蚁取食, 并且它们的种子能很快被蚂蚁 搬运走。

\section{3 蚂蚁的种类和数量}

在样地内共诱捕到蚂蚁 657 头, 其中全异巨首 蚁 394 头,占总数的 $60.0 \%$;黄立毛蚁 144 头,占 $21.9 \%$;梅花山大头蚁 53 头, 占 $8.1 \%$ 。其余是其 他种类的蚂蚁 (表 2)。由此可以说明全异巨首蚁 在桃金娘种子的搬运过程中起着最主要的作用。

\section{4 蚁巢种子调查}

全异巨首蚁和梅花山大头蚁的蚁巢里都没有桃 金娘的种子，所有䛎藏的桃金娘种子都放在巢口 种 子被从巢里运出来的泥土埋住。全异巨首蚁咜藏的 种子量大, 平均每个蚁巢种子陉藏量为 $195.0 \pm$ 82.9 粒 其中完好种子占 $73.2 \%$ 。破损的种子都是 种胚破损。梅花山大头蚁每个蚁巢平均咜藏桃金娘 种子 $28.8 \pm 11.4$ 粒,所有的种子都没有破损 (表 $3)$ 。

\section{5 桃金娘实生苗的调查}

在林下没发现有桃金娘实生苗 林窗、闲置的空 旷地、植物群落交错带都发现有桃金娘实生苗。在 桃金娘母树下没有实生苗; 距桃金娘母树树冠边缘 至少 $1.4 \mathrm{~m}$ 才有实生苗。在桃金娘母树附近的全异 巨首蚁和梅花山大头蚁蚁巢, 如果阳光充足则有实 生苗的存在。 
表 2 样地蚂蚁调查统计

Table 2 Species and number of the ants caught in the study plot

\begin{tabular}{lcc}
\hline \multicolumn{1}{c}{$\begin{array}{c}\text { 蚂蚁种类 } \\
\text { Species }\end{array}$} & $\begin{array}{c}\text { 蚂蚁数量 } \\
\text { Number of ants }\end{array}$ & $\begin{array}{c}\text { 占总个体数的百分比 } \\
\text { Percentage of total individuals }\end{array}$ \\
\hline 全异巨首蚁 Pheidologetion diversus & 394 & 60.0 \\
黄立毛蚁 Paratrechina flavipes & 144 & 21.9 \\
梅花山大头蚁 Pheidole meihuashanensis & 53 & 8.1 \\
聚纹双刺猛蚁 Diacamma rugosum & 7 & 1.1 \\
蚁亚科长结蚁属一种 Dolophra sp. & 39 & 5.9 \\
行军蚁亚科行军蚁属一种 Dorylus sp. & 20 & 3.0 \\
\hline
\end{tabular}

表 3 蚂蚁䛎藏桃金娘种子的数量

Table 3 Number of Rhodomyrtus tomentosa seeds stored in ant's nests

\begin{tabular}{lccc}
\hline $\begin{array}{c}\text { 蚂蚁种类 } \\
\text { Species }\end{array}$ & $\begin{array}{c}\text { 每巢种子数 } \\
\text { Seeds per nest (Mean } \pm \text { SE) }\end{array}$ & $\begin{array}{c}\text { 每巢完好种子数 } \\
\text { Intact seeds per nest }\end{array}$ & $\begin{array}{c}\text { 完好种子百分比 } \\
\text { Percentage of intact seeds }\end{array}$ \\
\hline 全异巨首蚁 Pheidologetion diversus & $195.0 \pm 82.9$ & $142.8 \pm 59.2$ & 73.2 \\
梅花山大头蚁 Pheidole meihuashanensis & $28.8 \pm 11.4$ & $28.8 \pm 11.4$ & 100.0 \\
\hline
\end{tabular}

\section{3 讨论}

桃金娘果实开始是青色 然后变成红色, 至成熟 时变成紫黑色 果实颜色的改变与周围绿色的环境 形成鲜明的对比,容易吸引鸟类受食。鸟类以果实 颜色为线索来搜寻果实, 特别是果实颜色经过两个 阶段的改变 能与周围的颜色形成鲜明的对比，使鸟 类更容易找到成熟的果实(Stiles ,1982)。实验统计 表明:有些鸟类喜食可溶性糖含量高、低蛋白质、高 脂肪的果肉 (Moermond \& Denslow,1989)。桃金娘 果实富含糖类和脂肪，能量价值高，容易被某些鸟取 食(刘芳和邓毓芳, 1997)。白头軒和红耳鸡是杂食 性鸟类，在中东和北非，鸺科鸟类是重要的种子传播 者 (Herrera ,1995)。在国内曾有报道白头棅鸟能传播 接骨草 (Sambucus chinensis)、樱桃 (Cerasus pseudocerasu)、乌柏 (Sapium rotundifolium) 的种子 红耳軾 是山黄麻 (Trema orientalis)、小果榕 (Ficus microcar$p a)$ 种子的传播者 (再景丞等, 1999; 黄双全和王孝 民 2000 汪直军等 2002)。食果鸟取食的方式和效 率取决于果实的大小和食果鸟体积大小, 尤其是鸟 的喙长和嘴宽 (Pedro,2000)。白头鹌的喙长为 11 - $18 \mathrm{~mm}$ 红耳軒喙长 12-20 mm (华南濒危动物研 究所,1988)。两种鸟类的口裂不适合桃金娘果实 的大小，不能整吞下果实，只能是豚食果实，但能吞 下种子。经显微镜下观察, 两种鸟类粪便中的桃金
娘种子并没有破损，可能是桃金娘种子种皮坚硬。 桃金娘果实成熟期长达 3 个月,为这两种鸺科的鸟 类提供了丰富的食源，其果实和种子是适应本地鸟 类传播的。但由于观察时间仅为桃金娘盛果期的 $5 \mathrm{~d}$ 时间, 时间相对较短，在鹤山是否还有其他鸟类 传播桃金娘种子还有待进一步的观察研究。

全异巨首蚁和梅花山大头蚁能够散布桃金娘种 子。蚁传播植物种子往往含有油质体结构 ( Pijl, 1982)。桃金娘种子没有油质体结构，但也被蚂蚁 搬运败藏。在搬运䛎藏的过程中，一部分种子被丢 弃在途中 其余的大部分种子也未被取食, 这都使种 子得到一定的散布。如全异巨首蚁在食果肉的过程 中用细泥把果实盖住, 这样可以为留在原地的种子 提供良好的萌发微环境。虽然全异巨首蚁也啃食一 部分桃金娘种子，但仍有 $73.2 \%$ 的种子没有受到影 响。这两种蚂蚁都能使桃金娘种子远离母树，尽量 散布到更多的地方, 降低了种子的聚集程度。而且 蚁丘也为种子提供了良好的微环境, 蚁丘土壤的通 气通水性好、酸碱度低、有机质含量高 ( 侯继华等, 2002 )。但只有阳光充足的蚁巢边才有实生苗的存 在。正如任海等 (1997) 指出 桃金娘不能在透光率 为 $32 \%$ 以下的林冠下正常生长并完成其更新过程。

表皮已破的桃金娘果实的果肉在当天被蚂蚁取 食 种子也被搬运。而表皮完好的桃金娘果实, 其果 肉在当天没有被蚂蚁取食 种子也没有被搬走, 但第 
二天早上这些果实就不见了。这些果实被何种动物 以何种方式吃掉或搬运有待进一步研究。这说明如 果食果鸟先取食, 被食果鸟啄破表皮落在地上的果 实容易被蚂蚁取食, 其种子也很快被搬运走。

我们研究的地区历史上曾为森林地带, 顶极群 落是亚热带季风常绿阔叶林。不断增长的人类活动 造成植被退化、水土流失、土壤癒薄和水源枯竭。人 为活动的干扰使许多地方退化为草坡 (彭少麟, 1996 )。桃金娘是华南地区退化荒坡上广为分布的 先锋种 (中国科学院华南植物研究所, 1956) 。白 头軒和红耳鸡吃完桃金娘果实后, 喜欢飞到较远较 空旷的高处、林窗以及植物群落交错带。这样可以 使桃金娘种子得到远距离传播,并且使种子扩散到 空旷处 增加桃金娘种子在这些地段土壤种子库中 的比例 ;同时空旷处、林窗、植物群落交错带的光照 充足, 适于种子萌发和幼苗生长, 使桃金娘能迅速生 长并形成群落。蚂蚁也在一定程度上使桃金娘种子 远离母树 散布到更远的地方, 为种子的萌发提供了 良好的微环境。所以在一定程度上, 食果鸟和蚂蚁 对桃金娘种子的传播有助于桃金娘占领受人为活动 干扰而退化为草坡的空旷地, 迅速成为先锋种。

\section{参考文献}

Cruz, A. 1981. Bird activity and seed dispersal of a montane forest tree (Dunalia arboresens) in Jamaica. Biotropica, 13 (Suppl. ) : $34-44$.

David, A. and Devon, L. 2000. Patterns of movement and seed dispersal of a tropical frugivore. Ecology, 122: 249 - 257.

Delectis Florae Reipublicae Popularis Sinicae Agendae Academiae Sinicae Edita(中国科学院中国植物志编辑委员会). 1984. Flora Reipublicae Popularis Sinicae(中国植物志). Science Press, Beijing, 121 -122. (in Chinese)

Edmund, W. 2000. Animal as dispersers. In: Michael, F. (ed. ), Seeds. CABI Publishing House, Oxford, 85 - 110.

Herrera, C. M. 1995. Plant-vertebrate seed dispersal systems in Mediterranean: ecological, evolutionary and historical determinants. Annual Review of Ecology and Systematics, 26: $705-727$.

Hou, J. H. (侯继华), Zhou, D. W. (周道玮) and Jiang, S. C. (姜世成). 2002. The effect of hill-building activities of ants on the species diversity of plant communities in Songnen Grassland. Acta Phytoecologica Sinica (植物生态 学报), 26: 323 - 329. (in Chinese with English abstract) Howe, H. F. and Smallwood, J. 1982. Ecology of seed dispersal. Annual Review of Ecology and Systematics, 13: 201
$-228$.

Huang, S. Q. (黄双全) and Wang, X. M. (王孝民). 2000. Seed dispersal by a frugivore Pycnonotus sinensis and the distribution of Sambucus chinensis. Acta Botanica Sinica( 植 物学报), 42: 1096 - 1100. (in Chinese with English abstract)

Hulme, P. E. 1998. Post-dispersal seed predation: consequences for plant demography and evolution. Perspectives in Plant Ecology, Evolution and Systematics, 1:32 - 46.

Jennifer, A. Z., Timothy, P. S. and Craig, R. A. 2001. Yellow jackets (Vespula spp.) disperse Trillium (spp.) seeds in eastern North America. American Midland Naturalist, 146: $444-446$.

Jose, L. V., Leonardo, G. and Gabriel, B. 2003. The beneficial effect of ants on the reproductive success of Dyckia floribunda (Bromeliaceae), an extrafloral nectary plant. Canadian Journal of Botany, 81:24-27.

Lin, Y. B. (林永标), Shen, W. J. (申卫军) and Peng, S. L. (彭少麟)。2003. Comparison of microclimatological effects of three plantations in Heshan of the lower subtropical, China. Acta Ecologica Sinica(生态学报), 23: 1657 1666. (in Chinese with English abstract)

Liu, F. (刘芳) and Deng, Y. F. (邓毓芳) . 1997. Preparation of Rhodomyrtus tomentosa Juice. Guangxi Forestry Science (广西林业科学), 26: $119-122$. (in Chinese)

Lu, C. H. (鲁长虎). 2003. Biology of mistletoe (Viscum coloratum) and its seed dispersal by frugivorous birds. Acta Ecologica Sinica(生态学报), 23: 834 - 839. ( in Chinese with English abstract)

McCall, C., Mitchell-Olds, T. and Waller, D. M. 1989. Fitness consequences of outcrossing in Impatiens capensis: tests of the frequency-dependent and sib-competition models. Evolution, 43: 1075 - 1084.

Moermond, T. C. and Denslow, J. S. 1985. Neotropical avian frugivores: patterns of behavior, morphology, and nutrition, with consequences for fruit selection. In: Buckley, P. A., Foster, M. S., Morton, E. S., Ridgely, R. S. and Buckley, F. G. (eds.), Neotropical Ornithology. American Ornithologist Union, Washington, 865 - 897.

Peres, C. A., Schiesari, L. C. and Dias-Leme, C. L. 1997. Vertebrate predation of Brazil-nuts (Bertholletia excesla, Lecythidaceae), an agouti-dispersed Amazonian seed crop: a test of the escape hypothesis. Journal of Tropical Ecology, 66: $708-720$.

Pedro, J. 2000. Fruits and frugivory. In: Michael, F. ( ed. ), Seeds. CABI Publishing House, Oxford, 125 - 165.

Peng, S. L. (彭少麟). 1996. The Dynamic of Forest Community in Low Subtropical Zone (南亚热带森林群落动态学). Science Press, Beijing, $24-29$. (in Chinese) 
Pijl, L. 1982. Principles of Dispersal in Higher Plants. Springer-Verlag, New York.

Ran, J. C. (再景丞), Xiong, Z. B. (熊志斌) and Zhang, C. G. (张丛贵). 1999. Preliminary study on the fruits eating birds on seed dispersal in Maolan Karst forest area. Journal of Guizhou Normal University( Natural Science) [ 贵州师范 大学学报 (自然科学版) ], 17:63 -66. (in Chinese with English abstract)

Ren, H. (任海), Peng, S. L. (彭少麟), Sun, G. C. (孙谷 畴) and Yu, Z. Y. (余作岳). 1997. The ecological comparison of Psychotria rubra and Rhodomyrtus tomentosa in South China. Acta Phytoecologica Sinica (植物生态学报), 21: 386 - 392. (in Chinese with English abstract)

Ron, J. and Thomas, G. 2002. Changes in interactions between juniper and mistletoe mediated by shared avian frugivores: parasitism to potential mutualism. Ecology, 130: $281-288$

South China Institute of Botany, Chinese Academy of Sciences (中国科学院华南植物研究所). 1956. Flora of Guangzhou (广州植物志). Science Press, Beijing, 200, 516.
( in Chinese)

South China Institute of Endangered Animals(华南濒危动物研 究所). 1988. Colorized Illustrated Handbook of Guangdong Birds (广东鸟类彩色图鉴). Guangdong Science and Technology Press, Guangzhou, 88 -89. (in Chinese)

Stiles, E. W. 1982. Fruit flags: two hypotheses. American Naturalist, 116: 670 - 688 .

Wang, Z. J. (王直军), Cao, M. (曹敏) and Li, G. F. (李国 锋). 2002. Trema orientalis seeds dispersed by birds and its ecological role. Zoological Research(动物学研究), 23: 214 -219. (in Chinese with English abstract)

Xiao, L. Y. (肖来云) and Pu, Z. H. (普正和) . 1994. Study on relationship between birds and Loranthaceae plants in Xishuangbanna, Yunnan Province. Acta Ecologica Sinica (生态学报), 14: 128 -134. (in Chinese with English abstract)

Zhang, Z. Y. (张智英), Cao, M. (曹敏) and Yang, X. D. (杨效东). 2001. Codariocalyx motorius seed removed by ants in Xishuangbanna. Acta Ecologica Sinica (生态学 报), 21: 1847 - 1853. (in Chinese with English abstract) 